Pacific Journal of Mathematics

HE RELATIONSHIP BETWEEN THE RADICAL OF A
LATTICE-ORDERED GROUP AND COMPLETE

Paul F. Conrad 


\title{
THE RELATIONSHIP BETWEEN THE RADICAL OF A LATTICE-ORDERED GROUP AND COMPLETE DISTRIBUTIVITY
}

\author{
PaUl ConRad
}

1. Introduction. Throughout this note let $G$ be a lattice-ordered group (notation 1-group). $G$ is said to be representable if there exists an 1-isomorphism of $G$ onto a subdirect sum of a cardinal sum of totally ordered groups (notation 0-groups). In particular, every abelian 1-group is representable. $G$ is said to be completely distributive if for $g_{i j} \in G$

$$
\bigwedge_{i \in I} \bigvee_{j \in J} g_{i j}=\bigvee_{f \in J I} \bigwedge_{i \in I} g_{i f(i)}
$$

provided the indicated joins and intersections exist.

For each $0 \neq g$ in $G$ let $R_{g}$ be the subgroup of $G$ that is generated by the set of all 1-ideals of $G$ not containing $g$. Then $R_{g}$ is an 1-ideal of $G$ and the radical of $G$ is defined to be

$$
R(G)=\cap R_{g} \quad(0 \neq g \in G) .
$$

In [2] it is shown that if $G$ is a divisible abelian 1-group, then there exists a minimal Hahn-type embedding of $G$ into an 1-group of real valued functions if and only if $R(G)=0$. Thus it would be useful to identify the class of abelian 1-groups with zero radicals, and to examine the properties of non-abelian 1-groups with zero radicals. In our main theorem we show that a representable 1-group $G$ is completely distributive if and only if $R(G)=0$. We also show $R(G)=0$ if and only if $G$ has a regular representation. This settles a question raised by Weinberg [6].

With no restrictions on $G$ we show that $R(G)$ is completely determined by the lattice $\mathscr{L}$ of all 1-ideals of $G$. In particular, if $G$ is a representable 1-group, then whether or not $G$ is completely distributive depends only on $\mathscr{L}$.

The author would like to express his gratitude to A. H. Clifford who read a rough draft of this note and made valuable suggestions. In particular, the present forms of Lemmas 1 and 2 are due to him.

2. Regular and essential $L$-ideals. If $g \in G$ and $M$ is an 1-ideal of $G$ that is maximal with respect to $g \notin M$, then $M$ is called a regular 1-ideal of $G$. Let $M^{*}$ be the intersection of all 1-ideals of $G$ that

Received June 6, 1963. This research was supported by a grant from the National Science Foundation. 
properly contain $M$. Then since $g \in M^{*}$, it follows that $M^{*}$ is the unique 1-ideal of $G$ that covers $M$. Let $\Gamma$ be an index set for the set of all pairs $\left(G^{\gamma}, G_{\gamma}\right)$ of 1-ideals of $G$ such that $G_{\gamma}$ is regular and $G^{\gamma}$ covers $G_{\gamma}$. Define $\alpha<\beta$ if $G^{\alpha} \subseteq G_{\beta}$. Then $\Gamma$ is a po-set, and we say that $\gamma \in \Gamma$ is a value of $g$ if $g \in G^{\gamma} \backslash G_{\gamma}$. In particular, the set of all values of $g$ is a trivially ordered subset of $\Gamma$. An element $\gamma \in \Gamma$ is called essential if there exists an $0 \neq h$ in $G$ such that all the values of $h$ are $\leqq \gamma$. In this case $G_{\gamma}$ is called an essential 1-ideal of $G$, and if $g \in G^{\gamma} \backslash G_{\gamma}$, then we say that $\gamma$ is an essential value of $g$.

Clearly the set $E$ of all essential elements in $\Gamma$ is a dual ideal of $\Gamma(\alpha<\beta \in \Gamma, \alpha \in E \rightarrow \beta \in E)$. The following lemma shows that the radical $R(G)$ of $G$ is completely determined by the essential ideals of $G$.

Lemma 1. The radical of $G$ is the intersection of essential 1-ideals of $G: \quad R(G)=\cap G_{\gamma}(\gamma \in E)$.

Proof. If $g \notin R(G)$, then $g \notin R_{h}$ for some $h$ in $G$ and by Zorn's lemma there exists an 1-ideal $M$ of $G$ that is maximal with respect to $g \notin M \supseteqq R_{h}$. Thus $M=G_{\gamma}$ for some $\gamma \in E, g \in G^{\gamma} \backslash G_{\gamma}$ and hence $g$ has an essential value. If $x \in \cap G_{\gamma}$, then $x$ has no essential value and hence $x \in R(G)$. Therefore $\cap G_{\gamma} \subseteq R(G)$. If $E$ is the null set, then $G=\cap G_{\gamma} \supseteqq R(G)$ and if $\gamma \in E$, then there exists $0 \neq h_{\gamma} \in G$ such that if $\delta$ is a value of $h_{\gamma}$, then $\delta \leqq \gamma$ and hence $G_{\delta} \leqq G_{\gamma}$. Thus $R_{h_{\gamma}} \leqq G_{\gamma}$ and so

$$
\bigcap_{\gamma \in E} G_{\gamma} \supseteqq \bigcap_{\gamma \in E} R_{h_{\gamma}} \supseteq \bigcap_{0 \neq g \in G} R_{g}=R(G)
$$

COROLlaRY. $R(G)=0$ if and only if each nonzero element in $G$ has at least one essential value.

We next show that $R(G)$ depends only on the lattice $\mathscr{L}$ of all 1-ideals of $G$. Note that a regular 1-ideal $M$ of $G$ is characterized by the fact that it is meet irreducible in $\mathscr{L}$. That is, if $M^{*}$ is the intersection of all 1-ideals of $G$ that properly contain $M$, then $M$ is properly contained in $M^{*}$.

Lemma 2. $\beta \in \Gamma$ is essential if and only if $\cap\left\{G_{\gamma}: \gamma \in \Gamma\right.$ and $\gamma \not \leq \beta\} \neq 0$.

Proof. Suppose that $0<h \in \cap\left\{G_{\gamma}: \gamma \in \Gamma\right.$ and $\gamma \not \equiv \beta$ and let $\alpha$ be a value of $h$. Then $h \notin G_{a}$ and so $\alpha \leqq \beta$. Thus all the values of $h$ are $\leqq \beta$, and hence $\beta$ is essential. Conversely assume that $G_{\beta}$ is essential and pick $0<h \in G$ such that all the values of $h$ are $\leqq \beta$. Then 
$h \in \cap\left\{G_{\gamma}: \gamma \in \Gamma\right.$ and $\gamma \not \equiv \beta$. For if $h \notin G_{\gamma}$, where $\gamma \not \equiv \beta$, then $h$ must have a value $\alpha \geqq \gamma$ which is impossible.

CoRollary. $R(G)$ is an invariant of the lattice $\mathscr{L}$ of all 1-ideals of $G$.

LemMa 3. For an 1-group $G$ the following are equivalent.

(1) $G / M$ is an 0-group for each regular 1-ideal $M$ of $G$.

(2) $G$ is representable.

Proof. For each $0 \neq g$ in $G$ pick an $l$-ideal $M_{g}$ of $G$ that is maximal with respect to not containing $g$. Then $\cap M_{g}=0$, and if (1) is satisfied, then each $G / M_{g}$ is an 0-group and the mapping of $x \in G$ upon $\left(\cdots, M_{g}+x, \cdots\right)$ is a representation of $G$. Conversely suppose that $G$ has a representation, then clearly

(3) if $a, b \in G^{+}$and $a \wedge b=0$, then $a \wedge(-x+b+x)=0$ for all $x \in G$. In fact, Sik [5] established that (2) and (3) are equivalent, but we only need that (2) implies (3). Let $M$ be an 1-ideal of $G$ that is maximal with respect to not containing $0<a \in G$, and let $A=M+a$. Suppose (by way of contradiction) that $G / M$ is not an 0 -group. Then there exist strictly positive elements $X$ and $Z$ in $G / M$ such that $X \wedge Z=M$.

Case I. $X \wedge A=M$. Then $P(A)=\{Y \in G / M:|Y| \wedge A=M\}$ is a convex 1-subgroup of $G / M$ that contains $X$ but not $A$. If $M<Y \in P(A)$, then $Y=M+y$, where $0<y \in G$, and $a=a \wedge y+a^{\prime}, y=a \wedge y+$ $y^{\prime}, a^{\prime} \wedge y^{\prime}=0$. Moreover

$$
M=A \wedge Y=M+a \wedge M+y=M+a \wedge y .
$$

Thus $a \wedge y \in M$ and so $Y=M+y^{\prime}$ and $A=M+a^{\prime}$. But by (3), $a^{\prime} \wedge\left(-g+y^{\prime}+g\right)=0$ for all $g$ in $G$ and hence $A \wedge-(M+g)+Y+$ $(M+g)=M$. Thus $P(A)$ is a nonzero 1-ideal of $G / M$ that does not contain $A$, and hence there exists an 1-ideal of $G$ that properly contains $M$ but not $\alpha$, but this contradicts the maximality of $M$.

Case II. $X \wedge A \neq M$. Then $P(X)$ is an 1-ideal of $G / M$ that contains $Z$ but not $A$, and once again we contradict the maximality of $M$. Therefore $G / M$ is an 0-group, and hence (2) implies (1).

CoRollary. If $G$ is representable and $R(G)=0$, then an element $g$ is positive in $G$ if and only $G_{\gamma}+g$ is positive for all essential values $\gamma$ of $g$.

Proof. If $g$ is positive in $G$, then $G_{\gamma}+g$ is positive for all values $\gamma$ of $g$, essential or otherwise. If $g$ is not positive, then $g=g \vee 0+$ 
$g \wedge 0=g^{+}+g^{-}$, where $g^{-} \neq 0$ and $g^{+} \wedge-g^{-}=0$. By the Corollary to Lemma 1 there exists an essential value $\gamma$ of $g^{-}$and by Lemma $3, G / G_{\gamma}$ is an 0 -group, and so $g^{+} \in G_{\gamma}$. Thus $\gamma$ is also an essential value of $g$ and $G_{\gamma}+g=G_{\gamma}+g^{-}$is negative.

LEMmA 4. If $0<g \in \vee A_{\lambda}$, where the $A_{\lambda}$ are 1-ideals of $G$, then $g=g_{1} \vee \cdots \vee g_{n}$, where $0 \leqq g_{i} \in \cup A_{\lambda}$ for $i=1, \cdots, n$.

Proof. This proof is due to T. Lloyd. Clearly $g=a_{1}+\cdots+a_{n}$, where the $a_{i} \in A_{\lambda i}$ for $i=1, \cdots, n$. Thus it suffices to show that $g \leqq a_{1}^{\prime} \vee \cdots \vee a_{n}^{\prime}$, where $a_{1}^{\prime} \in A_{\lambda i}$ for $i=1, \cdots, n$. For then

$$
\begin{aligned}
g & =\left(\left(a_{1}^{\prime} \vee 0\right) \wedge g\right) \vee \cdots \vee\left(\left(a_{n}^{\prime} \vee 0\right) \wedge g\right) \\
& =g_{1} \vee \cdots \vee g_{n}
\end{aligned}
$$

where $0 \leqq g_{i} \in A_{\lambda i}$ for $i=1, \cdots, n$. If $n=2$, then

$$
a_{1}+a_{2} \leqq 2 a_{1} \vee\left(a_{1}+a_{2}-a_{1}+a_{2}\right)=a_{1}^{\prime} \vee a_{2}^{\prime}
$$

because

$$
\begin{aligned}
0 \leqq\left|a_{1}-a_{2}\right| & =\left(a_{1}-a_{2}\right) \vee\left(a_{2}-a_{1}\right) \\
& =-a_{1}+\left(2 a_{1} \vee\left(a_{1}+a_{2}-a_{1}+a_{2}\right)\right)-a_{2} .
\end{aligned}
$$

Thus $a_{1}+\cdots+a_{n} \leqq\left(a_{1}+\cdots+a_{n-1}\right)^{\prime} \vee a_{n}^{\prime}$, and since $\left(a_{1}+\cdots+\right.$ $\left.a_{n-1}\right)^{\prime} \in \vee A_{\lambda i}(i=1, \cdots, n-1), \quad\left(a_{1}+\cdots+a_{n-1}\right)^{\prime}=b_{1}+\cdots+b_{n-1}$, where $b_{i} \in A_{\lambda i}$ for $i=1, \cdots, n-1$. Thus by induction $b_{1}+\cdots+b_{n-1} \leqq$ $a_{1}^{\prime} \vee \cdots \vee a_{n-1}^{\prime}$ and hence $g \leqq a_{1}^{\prime} \vee \cdots \vee a_{n}^{\prime}$.

3. Completely distributive $L$-groups. Let $A$ be a sublattice and and subdirect sum of a cardinal sum $B$ of 0 -groups $B_{\lambda}(\lambda \in A)$. If for each $\lambda$ in $\Lambda$, the projection $\rho_{\lambda}$ of $A$ onto $B_{\lambda}$ preserves infinite joins, then $A$ is called a regular subgroup of $B$. An 1-group $G$ is said to have a regular representation if it is 1-isomorphic to a regular subgroup of a cardinal sum of 0 -groups. It is easy to prove that an 1-group $G$ with a regular representation is completely distributive [6]. Weinberg has also shown ([6] Proposition 1.3) that the natural homomorphism of an 1-group $G$ onto $G / J$, where $J$ is an 1-ideal of $G$, preserves infinite joins if and only if $J$ is closed $\left(\vee j_{\lambda} \in G,\left\{j_{\lambda}: \lambda \in \Lambda\right\} \subseteq J \rightarrow \vee j_{\lambda} \in J\right)$. Thus it follows that $G$ has a regular representation if and only if there exists a family of closed 1-ideals $J_{\lambda}$ of $G$ such that $\cap J_{\lambda}=0$ and each $G / J_{\lambda}$ is an 0 -group.

Lemma 5. (Weinberg) An 1-group $G$ is completely distributive if and only if for each $0<g$ in $G$ there exists $0<g^{*}$ in $G$ such that

$$
g=\vee g_{\lambda}, g_{\lambda} \in G^{+} \rightarrow g^{*} \leqq g_{\lambda} \text { for some } \lambda .
$$


THEOREM. For a representable 1-group $G$ the following are equivalent.

(1) $R(G)=0$

(2) Each essential 1-ideal of $G$ is closed and $\cap G_{\gamma}=0(\gamma \in E)$.

(3) $G$ has a regular representation.

(4) $G$ is completely distributive.

Proof. By Lemma 3, for each $\gamma$ in $E, G / G_{\gamma}$ is an 0-group, and hence by the preceding discussion (2) implies (3) and (3) implies (4). Suppose that $G$ is completely distributive, and assume (by way of contradiction) that $0<g \in R(G)$. Then by Lemma 5 there exists $0<g^{*} \in G$ such that if $g=\vee g_{\alpha}\left(g_{\alpha} \in G^{+}\right)$, then $g^{*} \leqq g_{\alpha}$ for some $\alpha$. Since $g \in R(G)$ it follows that $g \in R_{g^{*}}=\vee A_{\lambda}$. where the $A_{\lambda}$ are the 1-ideals of $G$ not containing $g^{*}$. Thus by Lemma $4, g=g_{1} \vee \cdots \vee g_{n}$, where $0 \leqq g_{i} \in \cup A_{\lambda}$. But then $g^{*} \leqq g_{i}$ for some $i$, and hence $g^{*} \in \cup A_{\lambda}$ $a$ contradiction. Therefore (4) implies (1).

To complete the proof we must show that (1) implies (2). If (1) is satisfied, then by Lemma $1, \cap G_{\gamma}=0(\gamma \in E)$. Let $G_{\delta}$ be an essential 1 -ideal of $G$ and assume (by way of contradiction) that $G_{\delta}$ is not closed. Then there exists $g \in G^{+} \backslash G_{\delta}$ such that $g=\vee g_{j}\left(g_{j} \in G_{\delta}^{+}\right)$. Since $G_{\delta}$ is essential there exists $0<h \in G$ such that all the values of $h$ are $\leqq \delta$. We shall show that for some such $h, g-h \geqq g_{j}$ for all $j$, and hence $\vee g_{j}>\vee g_{j}-h=g-h \geqq \vee g_{j}$.

Case I. There exists $0<h \in G$ such that all the values of $h$ are $\leqq \delta$ and $G_{\delta}+h<G_{\delta}+g$. Since $g-h \notin G_{\delta}$ and $g_{j} \in G_{\delta}, g-h-g_{j} \neq 0$. By the Corollary to Lemma 3 it suffices to show that $G_{\beta}+g-h-g_{j}$ is positive for all values $\beta$ of $g-h-g_{j}$ in $E$. If $h \in G_{\beta}$, then $G_{\beta}+$ $g-h-g_{j}=G_{\beta}+g-g_{j}$ is positive. If $h \notin G_{\beta}$, then there exists a value $\gamma$ of $h$ such that $\gamma \geqq \beta$. But then $\beta \leqq \gamma \leqq \delta$, and since $g-h-g_{j} \in G^{\beta} \backslash G_{\delta}, \beta=\delta$. Therefore $G_{\beta}+g-h-g_{j}=G_{\delta}+g-h$ is positive.

Case II. For each $0<h \in G$ such that all of the values of $h$ are $\leqq \delta, G_{\delta}+h \geqq G_{\delta}+g$. If $\delta>\gamma \in E$, then we may choose $0<k \in G$ such that all of the values of $k$ are $\leqq \gamma<\delta$. But then $G_{\delta}+g>G_{\delta}=$ $G_{\delta}+k$. Therefore $\delta$ is minimal in $E$. If all values of $0<h$ are $\leqq \delta$, then $G_{\delta}+h \geqq G_{\delta}+g$ and so $G_{\delta}+g \wedge h=G_{\delta}+g$. If $\beta$ is a value of $g \wedge h$ in $E$, then $g \wedge h \in G^{\beta} \backslash G_{\beta}$ and hence $h \notin G_{\beta}$. Thus there exists a value $\gamma$ of $h$ such that $\beta \leqq \gamma \leqq \delta$ and since $\delta$ is minimal in $E, \beta=\delta$. Thus without loss of generality, $0<h \in G, \delta$ is the only value of $h$ in $E$ and $G_{\delta}+h=G_{\delta}+g$. If $g-h-g_{j} \neq 0$ and $\beta$ is a value of $g-h-g_{j}$ in $E$ then $h \in G_{\beta}$. Otherwise $\beta=\delta$, but $g-h-g_{j} \in G_{\delta}$. Therefore $G_{\beta}+g-h-g_{j}=G_{\beta}+g-g_{j}$ is positive for all values $\beta$ 
of $g-h-g_{j}$ in $E$. This completes the proof of our theorem. In proving that (4) implies (1) we did not use the hypothesis that $G$ is representable. Thus we have

CoRollary I. If $G$ is a completely distributive 1-group, then $R(G)=0$.

From the Corollary to Lemma 2 we have

COROLLARY II. If $G$ is a representable 1-group, then whether or not $G$ is completely distributive depends only on the lattice $\mathscr{L}$ of all 1-ideals of $G$.

4. Remarks and examples. Let $P$ be the 1-group of all order preserving permutations of the real line (with $f g(x)=f(g(x)$ ) and $f$ positive if $f(x) \geqq x$ for all $x$ ). Let

$A=\{f \in P: f$ induces the identity on $(-\infty, a]$ for some $a\}$, and

$B=\{f \in P: f$ induces the identity on $[a, \infty)$ for some $a\}$.

Let $C=A \cap B$. Then Holland [4] has shown that $A, B$ and $C$ are the only proper 1-ideals of $G$, and Higman [3] has shown that $C$ is algebraically simple. Therefore 0 is the only essential 1-ideal of $C$ and since $C / 0$ is not an 0 -group it follows from Lemma 3 that $C$ is not representable. Therefore $C$ satisfies property (2) of the theorem, but not property (3).

$(G, B)$ is the only value of each element in $A \backslash B$ and $(C, 0)$ is the only value of each nonzero element in $C$. Thus $B$ and 0 are essential 1-ideals of $P$, and in particular, $P$ satisfies (1). For each $n=1,2, \cdots$ let

$$
f_{n}(x)= \begin{cases}2 x & \text { if } x \leqq n \\ \frac{x+3 n}{2} & \text { if } n \leqq x \leqq 3 n \\ x & \text { if } 3 n \leqq x .\end{cases}
$$

Then $\left(\vee f_{n}\right)(x)=2 x$, and hence the $f_{n}$ belong to $B$, but $\vee f_{n} \notin B$. Therefore $P$ satisfies (1) but not (2).

A simple application of Lemma 5 shows that $P$ is completely distributive (or see [6] Example 3.3). Therefore (4) does not imply (2) or (3). On the other hand for arbitrary 1-groups, (3) $\rightarrow(2) \rightarrow(1)$. The remaining question is whether or not (1) or (2) implies (4) for non-representable 1-groups? Note that if $R(G)=0$ implies complete distributivity, then every 1-group with no proper 1-ideals is completely distributive, and in particular, every 1-group that is algebraically simple is completely distributive.

If the radical used in this note is replaced by one constructed in 
exactly the same way, but with 1 -ideals replaced by convex 1 -subgroups, then if this new radical is zero, the group is completely distributive. Also the new radical is an invariant of the lattice of all convex 1-subgroups of $G$. The proofs of these statements are analogous to those in this paper using the fact that if $C$ is a regular convex 1-subgroup, then the set of right cosets of $C$ in $G$ is totally ordered by

$$
C+x \leqq C+y \text { if } x \leqq y+c \text { for some } c \in C .
$$

Unfortunately the converse to the above is false. For example, the new radical for $P$ is $P$ itself and yet $P$ is completely distributive.

Let $G$ be an Archimedean 1-group. By Theorem 5.7 in [2], $R(G)=0$ if and only if $G$ has a basis, and by Theorem 7.3 in [1], $G$ has a basis if and only if $G$ is (isomorphic to) a subdirect sum of a cardinal sum of subgroups $R_{\gamma}$ of the reals which contains the finite cardinal sum of the $R_{\gamma}$. Thus we have a new proof for one of the main results in [6].

\section{REFERENCES}

1. P. Conrad, Some structure theorems for lattice-ordered groups, Trans. Amer. Math. Soc., 99 (1961), 212-240.

2. P. Conrad, J. Harvey and C. Holland, The Hahn embedding theorem for lattice-ordered groups, Trans. Amer. Math. Soc., 108 (1963), 143-169.

3. G. Higman, On infinite simple permutation groups, Publ. Math. Debrecen 3, (195354), 221-226.

4. C. Holland, The lattice-ordered group of automorphisms of an ordered set, Michigan Math. J., 10 (1963), 399-408.

5. F. Sik, Über Summen einfach geordneter Gruppen, Czech. Math. J., 8 (1958), 22-53.

6. E. C. Weinberg, Completely distributive lattice-ordered groups, Pacific J. Math., 12 (1962), 1131-1137. 



\title{
PACIFIC JOURNAL OF MATHEMATICS
}

\author{
EDITORS
}

\author{
Robert Osserman \\ Stanford University \\ Stanford, California \\ M. G. Arsove \\ University of Washington \\ Seattle 5, Washington
}

\author{
J. Dugundji \\ University of Southern California \\ Los Angeles 7, California \\ Lowell J. Paige \\ University of California \\ Los Angeles 24, California
}

\section{ASSOCIATE EDITORS}
E. F. BECKENBACH
B. H. NEUMANN
F. WOLF
K. YOSHIDA

\section{SUPPORTING INSTITUTIONS}

\author{
UNIVERSITY OF BRITISH COLUMBIA \\ CALIFORNIA INSTITUTE OF TECHNOLOGY \\ UNIVERSITY OF CALIFORNIA \\ MONTANA STATE UNIVERSITY \\ UNIVERSITY OF NEVADA \\ NEW MEXICO STATE UNIVERSITY \\ OREGON STATE UNIVERSITY \\ UNIVERSITY OF OREGON \\ OSAKA UNIVERSITY \\ UNIVERSITY OF SOUTHERN CALIFORNIA
}

\author{
STANFORD UNIVERSITY \\ UNIVERSITY OF TOKYO \\ UNIVERSITY OF UTAH \\ WASHINGTON STATE UNIVERSITY \\ UNIVERSITY OF WASHINGTON \\ AMERICAN MATHEMATICAL SOCIETY \\ CALIFORNIA RESEARCH CORPORATION \\ SPACE TECHNOLOGY LABORATORIES \\ NAVAL ORDNANCE TEST STATION
}

Mathematical papers intended for publication in the Pacific Journal of Mathematics should by typewritten (double spaced), and on submission, must be accompanied by a separate author's résumé. Manuscripts may be sent to any one of the four editors. All other communications to the editors should be addressed to the managing editor, L. J. Paige at the University of California, Los Angeles 24, California.

50 reprints per author of each article are furnished free of charge; additional copies may be obtained at cost in multiples of 50 .

The Pacific Journal of Mathematics is published quarterly, in March, June, September, and December. Effective with Volume 13 the price per volume (4 numbers) is $\$ 18.00$; single issues, $\$ 5.00$. Special price for current issues to individual faculty members of supporting institutions and to individual members of the American Mathematical Society: $\$ 8.00$ per volume; single issues $\$ 2.50$. Back numbers are available.

Subscriptions, orders for back numbers, and changes of address should be sent to Pacific Journal of Mathematics, 103 Highland Boulevard, Berkeley 8, California.

Printed at Kokusai Bunken Insatsusha (International Academic Printing Co., Ltd.), No. 6, 2-chome, Fujimi-cho, Chiyoda-ku, Tokyo, Japan.

PUBLISHED BY PACIFIC JOURNAL OF MATHEMATICS, A NON-PROFIT CORPORATION

The Supporting Institutions listed above contribute to the cost of publication of this Journal, but they are not owners or publishers and have no responsibility for its content or policies. 


\section{Pacific Journal of Mathematics}

\section{Vol. 14, No. $2 \quad$ June, 1964}

Tom M. (Mike) Apostol and Herbert S. Zuckerman, On the functional equation $F(m n) F((m, n))=F(m) F(n) f((m, n)) \ldots \ldots \ldots \ldots \ldots \ldots \ldots \ldots \ldots \ldots \ldots$

Reinhold Baer, Irreducible groups of automorphisms of abelian groups . . . . . . . 385

Herbert Stanley Bear, Jr., An abstract potential theory with continuous kernel . . . . 407

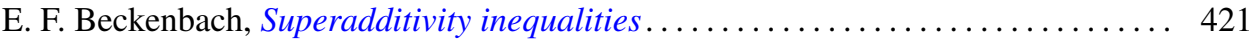

R. H. Bing, The simple connectivity of the sum of two disks . . . . . . . . . . . 439

Herbert Busemann, Length-preserving maps ...................... 457

Heron S. Collins, Characterizations of convolution semigroups of measures . . . . . . 479

Paul F. Conrad, The relationship between the radical of a lattice-ordered group and complete distributivity............................ 493

P. H. Doyle, III, A sufficient condition that an arc in $S^{n}$ be cellular . . . . . . . . . 501

Carl Clifton Faith and Yuzo Utumi, Intrinsic extensions of rings . . . . . . . . . . 505

Watson Bryan Fulks, An approximate Gauss mean value theorem . . . . . . . . . . 513

Arshag Berge Hajian, Strongly recurrent transformations . . . . . . . . . . . . . 517

Morisuke Hasumi and T. P. Srinivasan, Doubly invariant subspaces. II . . . . . . . 525

Lowell A. Hinrichs, Ivan Niven and Charles L. Vanden Eynden, Fields defined by

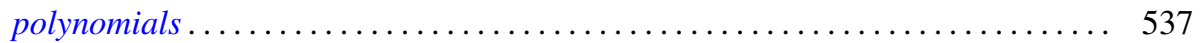

Walter Ball Laffer, I and Henry B. Mann, Decomposition of sets of group

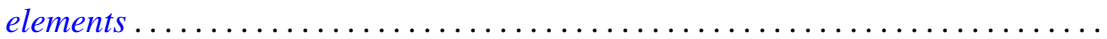

John Albert Lindberg, Jr., Algebraic extensions of commutative Banach

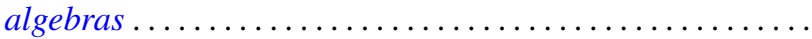

W. Ljunggren, On the Diophantine equation $C x^{2}+D=y^{n} \ldots$

M. Donald MacLaren, Atomic orthocomplemented lattices ....

Moshe Marcus, Transformations of domains in the plane and applications in the

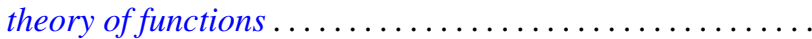

Philip Miles, $B^{*}$ algebra unit ball extremal points . .................. 627

W. F. Newns, On the difference and sum of a basic set of polynomials . . . . . . . 639

Barbara Osofsky, Rings all of whose finitely generated modules are injective ...... 645

Calvin R. Putnam, Toeplitz, matrices and invertibility of Hankel matrices . . . . . . . 651

Shoichiro Sakai, Weakly compact operators on operator algebras . . . . . . . . . 659

James E. Simpson, Nilpotency and spectral operators . . . . . . . . . . . . . 665

Walter Laws Smith, On the elementary renewal theorem for non-identically

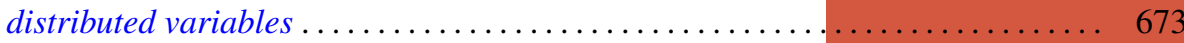

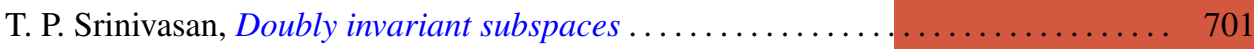

J. Roger Teller, On the extensions of lattice-ordered groups . . . . . . . . . . . . 709

Robert Charles Thompson, Unimodular group matrices with rational integers as

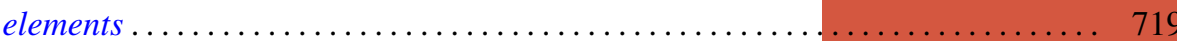

J. L. Walsh and Ambikeshwar Sharma, Least squares and interpolation in roots of unity

Charles Edward Watts, A Jordan-Hölder theorem .................... 731

Kung-Wei Yang, On some finite groups and their cohomology .............. 735

Adil Mohamed Yaqub, On the ring-logic character of certain rings ............ 741

Paul Ruel Young, A note on pseudo-creative sets and cylinders 